\title{
Radio Selection of the Most Distant Galaxy Clusters
}

Daddi, E.; Jin, S.; Strazzullo, V.; Sargent, M. T.; Wang, T.; Ferrari, C.; Schinnerer, E.;

Smolcic, V.; Calabro, A.; Coogan, R.; Delhaize, J.; Delvecchio, I.; Elbaz, D.; Gobat, R.; Gu, Q.; Liu, D.; Novak, M.; Valentino, F.

Published in:

Astrophysics Journal Letters

DOI:

10.3847/2041-8213/aa8808

Publication date:

2017

Document version

Publisher's PDF, also known as Version of record

Citation for published version (APA):

Daddi, E., Jin, S., Strazzullo, V., Sargent, M. T., Wang, T., Ferrari, C., Schinnerer, E., Smolcic, V., Calabro, A., Coogan, R., Delhaize, J., Delvecchio, I., Elbaz, D., Gobat, R., Gu, Q., Liu, D., Novak, M., \& Valentino, F. (2017). Radio Selection of the Most Distant Galaxy Clusters. Astrophysics Journal Letters, 846(2), [L31]. https://doi.org/10.3847/2041-8213/aa8808 


\title{
Radio Selection of the Most Distant Galaxy Clusters
}

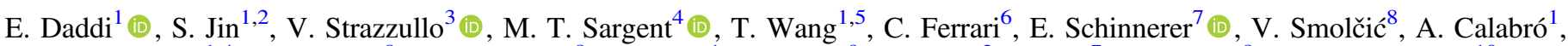 \\ R. Coogan ${ }^{1,4}$, J. Delhaize ${ }^{8}$, I. Delvecchio ${ }^{8}$, D. Elbaz ${ }^{1}$, R. Gobat ${ }^{9}$ (iD, Q. Gu ${ }^{2}$, D. Liu ${ }^{7}$, M. Novak ${ }^{8}$, and F. Valentino ${ }^{10}$ \\ ${ }^{1}$ CEA, IRFU, DAp, AIM, Université Paris-Saclay, Université Paris Diderot, Sorbonne Paris Cité, CNRS, F-91191 Gif-sur-Yvette, France \\ ${ }^{2}$ School of Astronomy and Space Science, Nanjing University, Nanjing 210093, China \\ ${ }^{3}$ Department of Physics, Ludwig-Maximilians-Universitat, Scheinerstr. 1, D-81679 Munchen, Germany \\ ${ }^{4}$ Astronomy Centre, Department of Physics and Astronomy, University of Sussex, Brighton BN1 9QH, UK \\ ${ }^{5}$ Institute of Astronomy, the University of Tokyo, and National Observatory Of Japan, Osawa, Mitaka, Tokyo 181-0015, Japan \\ ${ }^{6}$ Université Cote d'Azur, Observatoire de la Cote d'Azur, CNRS, Laboratoire Lagrange, Bd de l'Observatoire, CS 34229, F-06304 Nice cedex 4, France \\ Max Planck Institute for Astronomy, Konigstuhl 17, D-69117 Heidelberg, Germany \\ ${ }^{8}$ Department of Physics, Faculty of Science, University of Zagreb, Bijenička cesta 32, Zagreb 10000, Croatia \\ ${ }^{9}$ School of Physics, Korea Institute for Advanced Study, Hoegiro 85, Dongdaemun-gu, Seoul 02455, Korea \\ ${ }^{10}$ Dark Cosmology Centre, Niels Bohr Institute, University of Copenhagen, Juliane Maries Vej 30, DK-2100 Copenhagen, Denmark \\ Received 2017 July 26; revised 2017 August 22; accepted 2017 August 22; published 2017 September 8
}

\begin{abstract}
We show that the most distant X-ray-detected cluster known to date, $\mathrm{Cl} \mathrm{J1001} \mathrm{at} z_{\text {spec }}=2.506$, hosts a strong overdensity of radio sources. Six of them are individually detected (within $10^{\prime \prime}$ ) in deep 0 .' 75 resolution VLA $3 \mathrm{GHz}$ imaging, with $S_{3 \mathrm{GHz}}>8 \mu \mathrm{Jy}$. Of the six, an active galactic nucleus (AGN) likely affects the radio emission in two galaxies, while star formation is the dominant source powering the remaining four. We searched for cluster candidates over the full COSMOS $2 \mathrm{deg}^{2}$ field using radio-detected $3 \mathrm{GHz}$ sources and looking for peaks in $\Sigma_{5}$ density maps. $\mathrm{Cl} \mathrm{J1001}$ is the strongest overdensity by far with $>10 \sigma$, with a simple $z_{\text {phot }}>1.5$ preselection. A cruder photometric rejection of $z<1$ radio foregrounds leaves $\mathrm{Cl} \mathrm{J} 1001$ as the second strongest overdensity, while even using all radio sources $\mathrm{Cl}$ J1001 remains among the four strongest projected overdensities. We conclude that there are great prospects for future deep and wide-area radio surveys to discover large samples of the first generation of forming galaxy clusters. In these remarkable structures, widespread star formation and AGN activity of massive galaxy cluster members, residing within the inner cluster core, will ultimately lead to radio continuum as one of the most effective means for their identification, with detection rates expected in the ballpark of 0.1-1 per square degree at $z \gtrsim 2.5$. Samples of hundreds such high-redshift clusters could potentially constrain cosmological parameters and test cluster and galaxy formation models.
\end{abstract}

Key words: galaxies: clusters: general - galaxies: evolution - galaxies: formation - galaxies: high-redshift - radio continuum: galaxies

\section{Introduction}

The identification of the most distant, $z \gtrsim 2$ galaxy clusters -i.e., structures consistent with a single massive dark matter halo as opposed to megaparsec-scale loose overdensities like protoclusters (Diener et al. 2015) - is a very active topic of current research (see Overzier 2016 for a recent review). They represent the earliest generation of massive collapsed structures, progenitors to Coma-like clusters, and their abundance can constrain cosmological parameters. Also, they are unambiguous formation sites of massive ellipticals, and hence hold promise to shed light on the elusive processes that lead to the formation of passive, early-type galaxies. It is currently unclear if the morphological transformation of galaxies into spheroidal systems happens before or after entering the densest early cluster cores. The same holds for the quenching of star formation and passivization. High-redshift clusters are also interesting laboratories for studying (Valentino et al. 2016) the interaction between star formation/active galactic nucleus (AGN) activity and the hot intracluster medium (ICM), energy injection into the ICM, and its thermodynamical evolution. At redshifts $z \sim 1.5-3$ theory predicts that massive $\sim 10^{13-14} M_{\odot}$ dark matter halos should undergo a transition from being fed by cold streams to being shielded by a hot atmosphere that prevents refueling with fresh gas (Dekel et al. 2009). This process is not well constrained by theory. Observations are needed to trace the redshift and duration of this transition.
Evidence of persistent activity in massive structures at $z \sim 2-2.5$ suggests that this might occur later and/or at higher masses than currently expected (Valentino et al. 2015, 2016; Overzier 2016; Wang et al. 2016). Statistics from a larger number of clusters are required for definitive conclusions. Surveys aiming at detecting hot ICM, via X-rays or SunyaevZeldovich (SZ) emission, generally lack sensitivity for identifying clusters beyond $z \sim 2$. Clusters found approaching such a limit are so massive $\left(\gg 10^{14} M_{\odot}\right.$; Stanford et al. 2012; Newman et al. 2014) and evolved (i.e., dead) that they are far less interesting for the science discussed above. More typical $z \gtrsim 2$ systems have been discovered looking for concentrations of massive galaxies (e.g., Gobat et al. 2011, 2013; Yuan et al. 2014; Strazzullo et al. 2015; Wang et al. 2016), and X-ray emission searched for at their known positions, a posteriori.

Quite strikingly, the most distant X-ray-detected cluster known to date at $z=2.506$ (Wang et al. 2016, hereafter W16) displays a startling amount of star formation in its core $\left(L_{\mathrm{IR} 8-1000 \mu \mathrm{m}} \sim 2 \times 10^{13} L_{\odot}\right)$. Searching for concentrations of galaxy activity from star formation or AGNs might thus be a competitive means for finding the most distant structures. In this Letter, we explore this idea analyzing the W16 cluster radio continuum properties, keeping in mind that forthcoming deep radio surveys will cover large sky areas to remarkable depths. We use standard cosmology $(70,0.3,0.7)$ and a Chabrier IMF. 


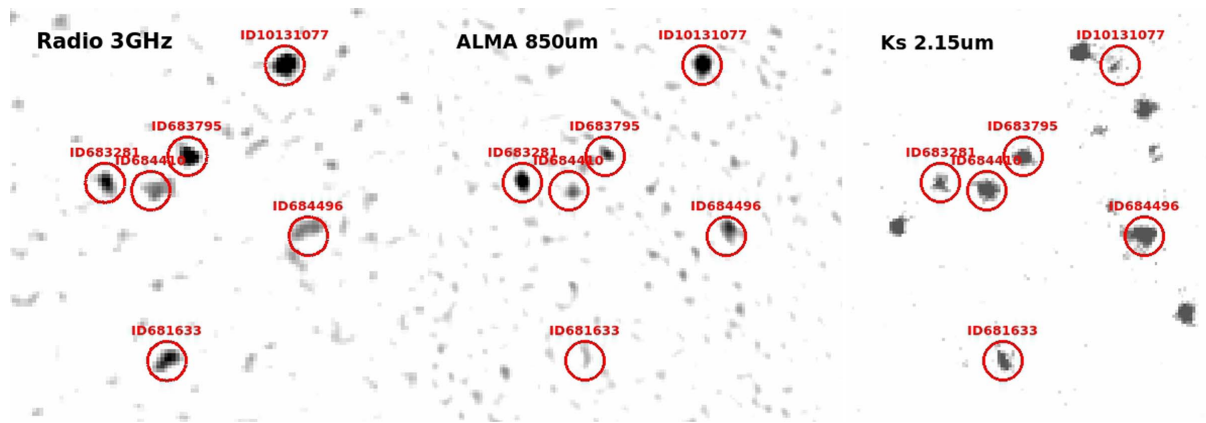

Figure 1. Maps of the W16 cluster region: VLA $3 \mathrm{GHz}$ (left), ALMA $850 \mu \mathrm{m}$ (center), and Ks-band (right), all $\sim 0$ !" 7 resolution. Panels are $20^{\prime \prime}$. Small circles ( $1^{\prime \prime}$ radius) help localize radio-detected cluster members (all spectroscopically confirmed; see Table 1).

\section{Radio Continuum Emission from Galaxies in $a=2.5$ Cluster}

Deep VLA observations of the COSMOS field at $3 \mathrm{GHz}$ have been obtained, fully reduced, analyzed, and publicly released by Smolčić et al. (2017). We have used the pointspread function (PSF) fitting technique and cataloging method of Liu et al. (2017) to obtain radio flux density measurements at $3 \mathrm{GHz}$ for all Ks-selected galaxies in the COSMOS2015 catalog of Laigle et al. (2016), supplemented with sources from Muzzin et al. (2013). Full details of this radio catalog together with the multi-wavelength properties in Herschel, Spitzer, SCUBA2, and other submillimeter probes of COSMOS galaxies will be presented in a forthcoming publication (S. Jin et al. 2017, in preparation). We concentrate here on the radio properties of galaxies in the surroundings of the W16 cluster, as compared to those in the full COSMOS $2 \mathrm{deg}^{2}$ field. Thanks to our PSF fitting technique we can push to deeper radio flux levels compared to blindly extracted signal-to-noise ratio $(\mathrm{S} / \mathrm{N})$ $>5$ catalogs in Smolčić et al. (2017), with high fidelity and completeness and with a very low expected spurious detection rate. Our simulations return typical rms sensitivities of 2.5-2.7 $\mu \mathrm{Jy}$ with well-behaved Gaussian-like uncertainties, close to the expected $2.3 \mu \mathrm{Jy}$ noise, allowing for reliable $\mathrm{S} / \mathrm{N}$ $>3$ detections at $3 \mathrm{GHz}$ down to about $8 \mu \mathrm{Jy}$. Radio sources without a Ks counterpart would be lost by our technique and are added back to the sample from the Smolčić et al. catalog (sources with multiple cataloged components, e.g., radio lobes, had been combined into one catalog entry).

Fitting a total of 589,713 priors, we obtain 17,803 radio continuum detections with $\mathrm{S} / \mathrm{N}>3$ in the COSMOS field, extracted over an area of $1.7 \mathrm{deg}^{2}$ from our Ks-band priors (doubling the number of detections at a fixed area, compared to the blind catalog). We find that six galaxies are radio detected within a $10^{\prime \prime}$ radius from the center ${ }^{11}$ of the W16 cluster; see Figure 1 and Table 1 . These are all spectroscopically confirmed cluster members and very red galaxies. The faintest, two MS galaxies, are not present in the Smolčić catalog. Four of the radio detections exhibit radio flux densities consistent with the FIR-radio correlation for star-forming galaxies at their redshift (Yun et al. 2001; Delhaize et al. 2017). Their radio emission is naturally explained by star formation alone. Two of these are relatively low mass while hosting high star formation rates (SFRs), i.e., they are starburst-like galaxies, probably mergerdriven. The other two are consistent with the main sequence at $z=2.5$ (see also W16). The remaining two detections have a

11 Average position of the six radio sources. radio flux excess over the SFRs inferred from the Atacama Large Millimeter/submillimeter Array (ALMA; Table 1; W16) by factors of $\gtrsim 3-5$ and do likely contain radio AGNs, with moderate intrinsic luminosities $\lesssim 10^{25} \mathrm{~W} \mathrm{~Hz}^{-1}$ (at rest $1.4 \mathrm{GHz}$, using also $1.4 \mathrm{GHz}$ data; Schinnerer et al. 2010). These two radio AGNs do not exibit any signatures of mid-IR or X-ray AGN activity (e.g., Delvecchio et al. 2017). The median ALMA $850 \mu \mathrm{m}$ to radio $3 \mathrm{GHz}$ flux ratio is $\sim 150$ (Table 1).

The total radio flux of the six cluster detections is $130 \mu \mathrm{Jy}$ with PSF fitting, or a maximum of $170 \mu \mathrm{Jy}$ when accounting for the possibly extended nature of some sources. Depending on the exact value, which radio-IR correlation we adopt, and factoring in possible AGN components, this corresponds to 20\%-90\% of the expectation from their Herschel+ALMA luminosities (W16). We radio-detect all sources producing the bulk of the SFR activity, with tentative evidence of a lower radio/ $\mathrm{L}_{\mathrm{IR}}$ ratio compared to the field.

Figure 2 shows distributions of photometric redshift, Ksband magnitudes, and radio fluxes. Based on KolmogorovSmirnov tests, the $z=2.5$ cluster galaxies are significantly fainter in Ks and at higher redshift than most radio detections above $8 \mu \mathrm{Jy}$ at $3 \mathrm{GHz}$. Instead, their radio flux density distribution, spanning $8-40 \mu \mathrm{Jy}$ (see Table 1 ), is still consistent with a random sampling of the parent catalog.

\section{Radio Selection of the $z=2.5$ W16 Cluster}

The six detections single out the W16 cluster as a very special environment in the radio, enough to allow its preselection as a cluster candidate even based on radio information alone. Such a selection can reach even stronger confidence when some low-level ancillary information is used in addition to radio, such as could be expected to be available in support of future generation, wide-area radio surveys. Following Figure 2, we considered the case of near-IR Ks-band imaging being available, or some moderate-quality photometric redshifts distinguishing $z>1.5$ sources, like the case for example with the BzK color preselection (Daddi et al. 2004) or red color selections (Franx et al. 2003; see also W16) or IRAC/ WISE red colors (e.g., Papovich et al. 2010). Finally, we also considered the direct use of COSMOS-quality photometric redshifts with an accuracy of about $7 \%$ in $(1+z)$ at $z \sim 2-3$ as available for W16 cluster galaxies.

As a first step, we compute the Poisson probabilities for chance associations of six galaxies within a $10^{\prime \prime}$ radius. This approach is approximative, given that the size and radius of the 
Table 1

Radio Detections in the W16 Cluster

\begin{tabular}{|c|c|c|c|c|c|c|c|c|c|c|c|}
\hline ID-Jin & $\begin{array}{l}\text { ID-COSMOS2015 } \\
\text { or Muzzin }\end{array}$ & $\begin{array}{l}\text { R.A. } \\
\text { deg }\end{array}$ & $\begin{array}{c}\text { Decl. } \\
\text { deg }\end{array}$ & $\underset{\|}{\underset{\prime}{\text { Distance }}}$ & $z_{\text {phot }}$ & $z_{\text {spec }}$ & $\begin{array}{c}\log M^{*} \\
M_{\odot}\end{array}$ & $\begin{array}{c}S_{3 \mathrm{GHz}} \\
\mu \mathrm{Jy}\end{array}$ & $\begin{array}{c}S_{850 \mu \mathrm{m}} \\
\mathrm{mJy}\end{array}$ & $\begin{array}{c}\mathrm{Ks} \\
\mathrm{AB} \mathrm{mag}\end{array}$ & Origin of Radio \\
\hline 681633 & 130651 & 150.2389798 & 2.3339305 & 8.5 & 2.69 & $2.50 \pm 0.02$ & 11.18 & $27.6 \pm 2.7$ & $<1.2(3 \sigma)$ & 22.43 & AGN \\
\hline 683281 & 130891 & 150.2398843 & 2.3364591 & 4.9 & 2.74 & 2.513 & 11.33 & $20.6 \pm 2.5$ & $3.77 \pm 0.32$ & 22.56 & SB \\
\hline 683795 & 130933 & 150.2387007 & 2.3368268 & 2.2 & 2.29 & 2.500 & 11.02 & $24.1 \pm 2.7$ & $1.66 \pm 0.21$ & 22.31 & AGN \\
\hline 684410 & 130901 & 150.2392700 & 2.3363813 & 2.7 & 2.36 & 2.508 & 11.23 & $8.8 \pm 2.9$ & $2.23 \pm 0.41$ & 21.7 & MS \\
\hline 684496 & 130949 & 150.2370141 & 2.3357152 & 5.8 & 2.26 & 2.503 & 11.32 & $10.8 \pm 3.2$ & $1.69 \pm 0.25$ & 20.93 & MS \\
\hline 10131077 & 131077 & 150.2373500 & 2.3381379 & 8.0 & 2.82 & 2.494 & 10.92 & $39.5 \pm 3.1$ & $5.26 \pm 0.26$ & 24 & SB \\
\hline
\end{tabular}

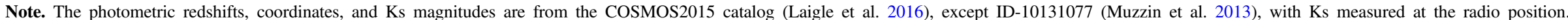

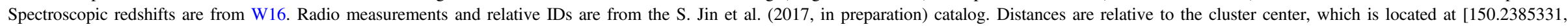
2.3362418]. For the "Origin of Radio" column see the discussion in the text. 

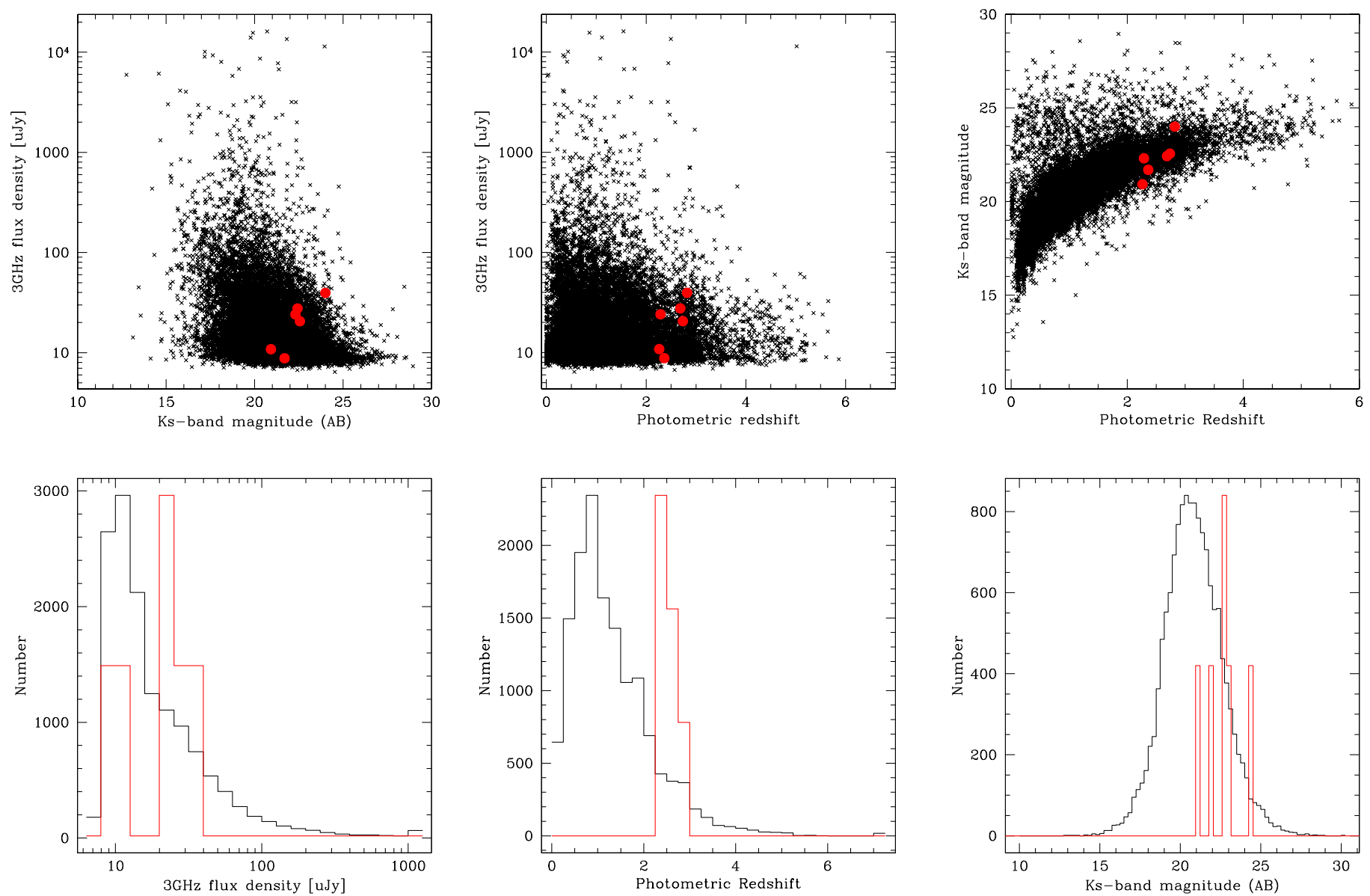

Figure 2. Observed properties, including radio fluxes, Ks-band magnitudes, and photometric redshifts, of the radio detections in the W16 cluster (red) compared to the parent sample of all $3 \mathrm{GHz}$ detections in COSMOS. Red histograms values are arbitrarily scaled to fit the plot.

search area were chosen ad hoc, although we note that the $10^{\prime \prime}$ radius corresponds to the core radius of a massive $\sim 10^{14} M_{\odot}$ cluster at $z>2$ (W16). More importantly, this approach neglects clustering, i.e., the presence of galaxies in smaller structures, filaments, and even projected random association of physical galaxy pairs, that can conspire to increase the spurious (non-single cluster) occurrence of a large counting event. This can nevertheless provide a useful starting guide. The Poisson probability of a W16-like association is fairly low even when just considering radio sources: 0.02 events are expected in COSMOS. This decreases to $2.0 \times 10^{-4}$ considering only Ksfaint radio sources, and to $10^{-5}$ and $10^{-7}$ for sources with $z_{\text {phot }}>1.5$ and $2<z_{\text {phot }}<3$, respectively.

A more objective approach is to use $\Sigma_{5}$ peak statistics ${ }^{12}$ to search for overdensities in the full COSMOS field, following the method from Strazzullo et al. (2015), which allows for a derivation of the dispersion and thus for an evaluation of their significance. Figure 3 shows the resulting $\Sigma_{5}$ maps and distribution of $\Sigma_{5}$ readings over the full COSMOS areas for the various selections described in Table 2 . When allowing for a photometric redshift preselection of high-redshift radio sources, the W16 cluster is recovered as the first ranked overdensity. Pre-selecting radio sources with $2<z<3$, the

\footnotetext{
${ }^{12} \Sigma_{5}=5 /\left(\pi R_{5}^{2}\right)$, where $R_{5}$ is the distance to the fifth closest neighbor. This approach as well requires choosing, somewhat arbitrarily, a number (5). An alternative approach would be convolving the catalog with a 2D profile as expected for a cluster; see Figure 9 in W16. We find this leads to consistent results.
}

W16 cluster is a $12.3 \sigma$ overdensity, long ahead of the second ranking object, seen with $6.5 \sigma$ at position [149.86924, $2.3417165]$. With a cruder $z_{\text {phot }}>1.5$ constraint, as obtainable from color selections, the W16 cluster is detected at $>10 \sigma$. The second ranking cluster is again detected with far less significance at $7.6 \sigma$ near $[150.31505,2.7126151]$. This latter structure seems loose and possibly the result of line-of-sight alignments. Using all Ks-faint radio sources returns the W16 cluster as the second ranking overdensity with $8.7 \sigma$, after a $9.2 \sigma$ at $[150.14933,2.5922460]$ (however, the latter is likely a chance alignment including low- $z$ sources). When using all radio-detected sources with $\mathrm{S} / \mathrm{N}>3$ the W16 is found as the fourth ranked $(7.7 \sigma)$, after three more $7.8-8.1 \sigma$ peaks [150.14933, 2.5933659]-[150.00879,2.2741797]-[149.83185, 2.5704069]. All of the latter appear to be chance alignments, judging from their $z_{\text {phots }}$, except perhaps a possibly genuine but loose concentration at $z \sim 0.9$ in the latter case. A detailed discussion of the nature of these possibly significant $\Sigma_{5}$ peak is beyond the scope of this Letter, where we concentrate on the well-studied case of the spectroscopically confirmed $z=2.506$ cluster W16.

We caution that with six sources only and with two close to the limit of our current radio photometry, the W16 cluster provides a highly significant but perhaps fragile detection. One could imagine that out of many realizations of a similar dark matter halo structure at $z=2.5$, at times, one or two galaxies could remain below current detection limits (for effects of noise, lower SFR, lower AGN activity), preventing detection in 

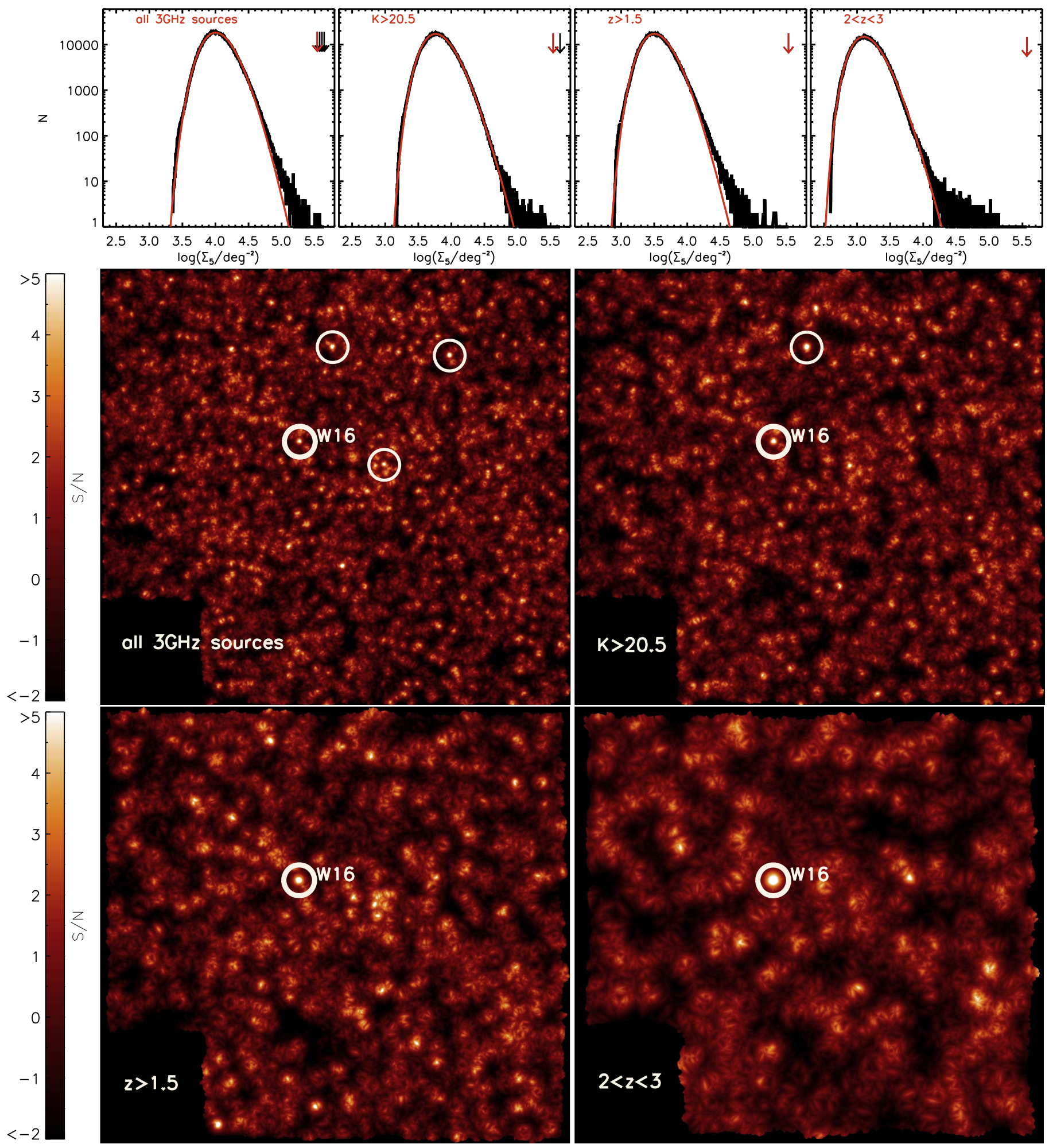

Figure 3. $\Sigma_{5}$ maps (bottom four panels) and distributions of readings (top four panels), for the sample selections discussed in the text. The W16 cluster is shown with a thicker white circle uniquely identified in the 2 bottom maps. The bottom left corner of each map is not analyzed due to poor coverage in the Ks-band. The histograms are fitted with $\Gamma$ distributions. Arrows show detected overdensities down to the W16 cluster (red). Figure 1 of W16 provides a useful comparison of a similar diagnostic on Ks-selected galaxies.

the radio in presence of low number statistics. Those four of the six $\mathrm{W} 16$ radio detections that appear in the Smolčić et al. $3 \mathrm{GHz}$ catalog (i.e., have $S_{3 \mathrm{GHz}}>20 \mu \mathrm{Jy}$ ) still define an $\sim 11 \sigma$ overdensity (when adopting, ad hoc, the $\Sigma_{4}$ statistics).

\section{Discussion}

We have demonstrated above that using only radio-detected sources, ideally with alternative information in the form of photometric redshifts or even single-band photometry, allows 
Table 2

Evaluation of Overdensity of the W16 Cluster from Radio Plus Ancillary Selections

\begin{tabular}{|c|c|c|c|c|c|c|}
\hline Selection & Full sample & $\#$ in $R=10^{\prime \prime}(1)$ & $P(\geqslant 6)(2)$ & Det. Rate(3) & $\Sigma_{5}$ Peak Rank & Comments(4) \\
\hline $\mathrm{S} / \mathrm{N}_{3 \mathrm{GHz}}>3$ & 17803 & 0.25 & $3.0 \times 10^{-7}$ & 0.02 & 4 & $7.6 \sigma$, after 3 with $7.8-8.1 \sigma$ \\
\hline $\mathrm{S} / \mathrm{N}_{3 \mathrm{GHz}}>3 \& K s>20.5$ & 8016 & 0.11 & $2.8 \times 10^{-9}$ & $2.0 \times 10^{-4}$ & 2 & $8.7 \sigma$, after a $9.2 \sigma$ \\
\hline $\mathrm{S} / \mathrm{N}_{3 \mathrm{GHz}}>3 \& z_{\text {phot }}>1.5$ & 4952 & 0.07 & $1.6 \times 10^{-10}$ & $10^{-5}$ & 1 & $10.0 \sigma$, the second is at $7.6 \sigma$ \\
\hline $\mathrm{S} / \mathrm{N}_{3 \mathrm{GHz}}>3 \& 2<z_{\text {phot }}<3$ & 1994 & 0.03 & $1.4 \times 10^{-12}$ & $10^{-7}$ & 1 & $12.3 \sigma$, the second is at $6.5 \sigma$ \\
\hline
\end{tabular}

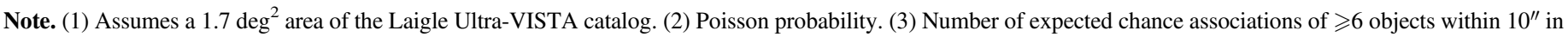

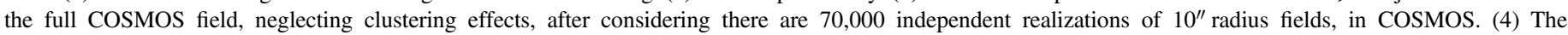

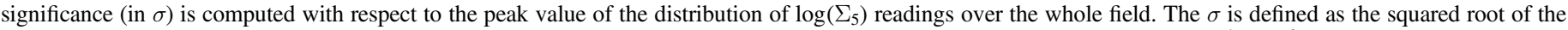

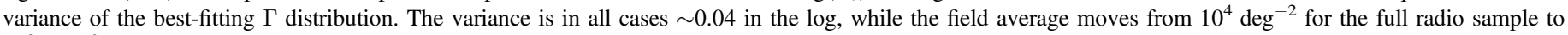
$10^{3} \mathrm{deg}^{-2}$ for the $2<z_{\text {phot }}<3$ case (see also Figure 3, the average separation changing by a factor of 3 as a result of the changing number density).

us to pinpoint the W16 cluster. This is quite promising news for future large area radio surveys, which will reach very faint flux densities (Padovani 2016). For example, even in phase1, SKA will likely carry out a full-sky survey to few $\mu \mathrm{Jy}$ sensitivity and with $\sim 2^{\prime \prime}$ resolution at $1-2 \mathrm{GHz}$, while covering $\sim 1000$ $\mathrm{deg}^{2}$ to $\sim 1 \mu \mathrm{Jy}$ depth and subarcsec resolution (Prandoni \& Seymour 2015), surpassing the depth and sensitivity of our $3 \mathrm{GHz}$ VLA data, allowing us in principle to find W16-like clusters over the whole sky, and perhaps much further away and in great numbers. Current plans also envisage excellent prospects for ngVLA, when observing at $\sim 2-3 \mathrm{GHz}$ (Selina \& Murphy 2017). Our work identifies important synergies between future radio, optical, NIR, and X-ray surveys. The community preparing future multiband facilities is perfectly aware of the necessity of this joint effort, which therefore starts to be discussed and organized (see, e.g., Table 5 in Prandoni \& Seymour 2015; see also Bacon et al. 2015, LSST versus SKA; Ciliegi \& Bardelli 2015; Kitching et al. 2015, Euclid versus SKA). For example, Euclid full-sky photometry (compare to Figure 2) already should be sufficient to reproduce the different galaxy selections that we explored in this work, thus likely greatly enhancing the contrast toward genuine high-redshift cluster structures found in the radio. On the other hand, radio surveys over large areas but with shallower depth and/or lower angular resolution than the $3 \mathrm{GHz}$ COSMOS data (we recall that we have 0.75 resolution and $2.3 \mu \mathrm{Jy} \mathrm{rms}$ at $3 \mathrm{GHz}$, equivalent to $4.3 \mu \mathrm{Jy}$ at $1.4 \mathrm{GHz}$ for $\alpha=0.8$ ) like the EMU survey with ASKAP at $1.4 \mathrm{GHz}$, LOFAR low frequency surveys, and even MIGHTEE from MeerKAT-see details in Norris et al. (2013) for all of these-would be substantially less efficient in finding $z>2.5-3$ clusters.

ALMA interferometric imaging (Figure 1 and Table 1) will provide efficient screening of radio-selected high- $z$ cluster candidates, confirming the high galaxy SFRs and their distances (the radio to submillimeter flux ratio is a rough redshift indicator; Carilli \& Yun 1999). The W16 cluster is the strongest SPIRE source in the Herschel imaging of CANDELS-COSMOS. While from Herschel alone it is impossible to know if that corresponds to a single bright starburst or multiple sources, this is within the reach of ALMA that can deliver subarcsec resolution in the submillimeter. With less than one minute per field at $345 \mathrm{GHz}$ ALMA can nowadays reach deeper than the data presented in Table 1 and Figure 1, making ALMA pre-screening of high- $z$ cluster candidates from radio surveys viable even for large samples of cluster candidates. The $20^{\prime \prime}$ ALMA field of view at $345 \mathrm{GHz}$ is well matched to the size of the overdensities, and W16 already demonstrated that millimeter spectroscopy with NOEMA/ALMA/VLA is a competitive means to spectroscopically confirm the clusters in a single shot. On the other hand, ALMA cannot perform blind cluster searches on large areas. Observations at lower frequencies in the radio have substantial advantages with much wider fields of view.

Overall, it appears that the reason why there is such a strong contrast in radio for the W16 cluster is twofold: first, the overall strong rise of specific SFR to high-z (e.g., Schreiber et al. 2015; Faisst et al. 2016) with its accompanying AGN activity (e.g., Mullaney et al. 2012) implies fairly high radio fluxes for massive star-forming galaxies. But even more crucially, the vast majority $(>80 \%)$ of massive galaxies in the W16 cluster core are strongly star-forming $\left(\mathrm{SFR} \gtrsim 100 M_{\odot} \mathrm{yr}^{-1}\right.$ ) rather than passive (the opposite is seen even in the $z \sim 2$ cluster $\mathrm{Cl} 1449$; Gobat et al. 2013; Strazzullo et al. 2013; see also Newman et al. 2014). This appears to be a genuine transition to what could be objectively defined as a star-forming cluster. Such star-forming clusters would be deliverable in large numbers by radio preselection in future radio surveys, as advocated here. As discussed in the Introduction, these would be some of the most interesting places to study early galaxy formation and evolution in cluster structures, with the cluster red sequence still in the process of birth at $z \sim 2$ (Strazzullo et al. 2016), and the interplay between ICM and galaxy formation (Valentino et al. 2016). Quite interestingly, visual inspection suggests elongated radio morphology (misaligned with the optical) in three of the six detections in the W16 cluster (IDs: 681633, AGN; 684496, SF; 684410, SF; see Figure 1), hinting at the possibility that small-scale radio jets might be present in half of the sources. Radio might thus provide insights into cluster physics and interaction between galaxies and the ICM. SKA1 band 2 should provide 0 ". 4 resolution and deeper data over an area $\times 1000$ times larger than COSMOS and will clarify this issue.

The W16 cluster recovery performance obtainable from radio samples is quite comparable to that achievable using $J-K s$ color on Ks-selected samples that returned $11.6 \sigma$ (W16), with respect to $12.3 \sigma$ here, both first rank. The two techniques are complementary and could beneficially be used together: the advantage of the radio is that it is insensitive to dust obscuration and directly selects on the galaxy star formation activity (and AGN emission adds on top of that), thus pinpointing forming/active clusters, while a pure selection on optical colors could potentially reveal old and dead clusters as well. This approach is also quite different from the use of very luminous radio-galaxies as massive beacons to locate distant clusters (e.g., Miley et al. 2006; Miley \& De Breuck 2008; Galametz et al. 2012; Cooke et al. 2015), but future large area radio surveys might combine the 
two efforts, allowing one to select at the same time the rare ultra-bright radio-galaxies as well as the faint cluster galaxies, thus clarifying the cosmic relevance of (proto)clusters around radio-galaxies. While subject to similar limitations as Herschel searches, Planck Collaboration XXXVII (2015) and Planck Collaboration XXXIX (2016) selected large samples of high-redshift protocluster candidates. However, these candidates likely mostly comprise chance alignments of smaller structures at different redshifts (Negrello et al. 2017).

Based on the recovery of a single W16-like cluster in the COSMOS field, we expect a return of $0.1-1 \mathrm{deg}^{-2}$ W16-like clusters at the $68 \%$ confidence level (Poisson counts). Even limiting the SKA1 survey to over $1000 \mathrm{deg}^{2}$ could thus produce between 100-1000 $z \gtrsim 2.5$ clusters. The number density of similarly massive halos at $z>3.5$ would be about $\times 10$ smaller, still allowing sizable samples of up to $10-100$. At $z=3.5$ we would expect $\times 2$ smaller radio fluxes compared to $z=2.5$ at fixed observing frequency and luminosity. This could be mitigated by the expected increase of the specific SFR with redshift, and the SKA1 will reach deeper than our current data.

As discussed in W16, the identification of this structure in a field like COSMOS is somewhat in tension with $\Lambda \mathrm{CDM}$, suggesting that actual returns will be toward the lower range, or the mass of the W16 system has been overestimated. However, expected numbers are large enough that future samples of high-redshift clusters might allow interesting tests of cosmology, in addition to cluster and galaxy formation physics, given that Athena will have the sensitivity to constrain their dark matter halo masses from the X-rays (e.g., Padovani et al. 2017).

S.J. acknowledges Chinese Scientific Council funding. M.T.S. was supported by a Royal Society Leverhulme Trust Senior Research Fellowship (LT150041). We acknowledge European Union's Horizon 2020 support (ERC 694343: E.S., D.L.; ERC 337595: V.S., J.D., I.D., M.N.). Q.G. acknowledges the National Key Research and Development Program of China (No. 2017YFA0402703).

\section{ORCID iDs}

E. Daddi (iD https://orcid.org/0000-0002-3331-9590

V. Strazzullo (1) https://orcid.org/0000-0001-7975-2894

M. T. Sargent (1D https://orcid.org/0000-0003-1033-9684

E. Schinnerer (iD https://orcid.org/0000-0002-3933-7677

R. Gobat (i) https://orcid.org/0000-0003-0121-6113

\section{References}

Bacon, D., Bridle, D., Abdalla, F. B., et al. 2015, in Proc. of Advancing Astrophysics with the Square Kilometre Array (AASKA14) (Trieste: SISSA), 145

Carilli, C. L., \& Yun, M. S. 1999, ApJL, 513, L13

Ciliegi, P., \& Bardelli, S. 2015, in Proc. of Advancing Astrophysics with the Square Kilometre Array (AASKA14) (Trieste: SISSA), 150

Cooke, E. A., Hatch, N. A., Rettura, A., et al. 2015, MNRAS, 452, 2318

Daddi, E., Cimatti, A., Renzini, A., et al. 2004, ApJ, 617, 746

Dekel, A., Birnboim, Y., Engel, G., et al. 2009, Natur, 457, 451

Delhaize, J., Smolčić, V., Delvecchio, I., et al. 2017, A\&A, 602, A4

Delvecchio, I., Smolčić, V., Zamorani, G., et al. 2017, A\&A, 602, A3

Diener, C., Lilly, S. J., Ledoux, C., et al. 2015, ApJ, 802, 31

Faisst, A. L., Capak, P., Hsieh, B. C., et al. 2016, ApJ, 821, 122

Franx, M., Labbé, I., Rudnick, G., et al. 2003, ApJL, 587, L79

Galametz, A., Stern, D., De Breuck, C., et al. 2012, ApJ, 749, 169

Gobat, R., Daddi, E., Onodera, M., et al. 2011, A\&A, 526, A133

Gobat, R., Strazzullo, V., Daddi, E., et al. 2013, ApJ, 776, 9

Kitching, T., Bacon, D., Brown, M., et al. 2015, in Proc. of Advancing Astrophysics with the Square Kilometre Array (AASKA14) (Trieste: SISSA), 146

Laigle, C., McCracken, H. J., Ilbert, O., et al. 2016, ApJS, 224, 24

Liu, D., Daddi, E., Dickinson, M., et al. 2017, ApJ, submitted (arXiv:1703. 05281)

Miley, G., \& De Breuck, C. 2008, A\&ARv, 15, 67

Miley, G. K., Overzier, R. A., Zirm, A. W., et al. 2006, ApJL, 650, L29

Mullaney, J. R., Daddi, E., Béthermin, M., et al. 2012, ApJL, 753, L30

Muzzin, A., Marchesini, D., Stefanon, M., et al. 2013, ApJ, 777, 18

Negrello, M., Gonzalez-Nuevo, J., De Zotti, G., et al. 2017, MNRAS, 470,2253

Newman, A. B., Ellis, R. S., Andreon, S., et al. 2014, ApJ, 788, 51

Norris, R. P., Afonso, J., Bacon, D., et al. 2013, PASA, 30, e020

Overzier, R. A. 2016, A\&ARv, 24, 14

Padovani, P. 2016, A\&ARv, 24, 13

Padovani, P., Combes, F., Diaz Trigo, M., et al. 2017, arXiv:1705.06064

Papovich, C., Momcheva, I., Willmer, C. N. A., et al. 2010, ApJ, 716, 1503

Planck Collaboration XXXIX 2016, A\&A, 596, A100

Planck Collaboration XXXVII 2015, A\&A, 582, A30

Prandoni, I., \& Seymour, N. 2015, in Proc. of Advancing Astrophysics with the Square Kilometre Array (AASKA14) (Trieste: SISSA), 67

Schinnerer, E., Sargent, M. T., Bondi, M., et al. 2010, ApJS, 188, 384

Schreiber, C., Pannella, M., Elbaz, D., et al. 2015, A\&A, 575, A74

Selina, R., \& Murphy, E. 2017, NRAO ngVLA Technical Memo 17, http:// library.nrao.edu/public/memos/ngvla/NGVLA_17.pdf

Smolčić, V., Novak, M., Bondi, M., et al. 2017, A\&A, 602, A1

Stanford, S. A., Brodwin, M., Gonzalez, A. H., et al. 2012, ApJ, 753, 164

Strazzullo, V., Daddi, E., Gobat, R., et al. 2015, A\&A, 576, L6

Strazzullo, V., Daddi, E., Gobat, R., et al. 2016, ApJL, 833, L20

Strazzullo, V., Gobat, R., Daddi, E., et al. 2013, ApJ, 772, 118

Valentino, F., Daddi, E., Finoguenov, A., et al. 2016, ApJ, 829, 53

Valentino, F., Daddi, E., Strazzullo, V., et al. 2015, ApJ, 801, 132

Wang, T., Elbaz, D., Daddi, E., et al. 2016, ApJ, 828, 56 (W16)

Yuan, T., Nanayakkara, T., Kacprzak, G. G., et al. 2014, ApJL, 795, L20

Yun, M. S., Reddy, N. A., \& Condon, J. J. 2001, ApJ, 554, 803 